\title{
Pathogenic Variation in Spore Populations of Sporisorium scitamineum, Causal Agent of Sugarcane Smut in Australia
}

\author{
Shamsul A. Bhuiyan and Barry J. Croft, Sugar Research Australia (SRA), Woodford, QLD 4514 Australia; and Joanne K. Stringer \\ and Emily C. Deomano, SRA, Indooroopilly QLD 4068 Australia
}

\begin{abstract}
Bhuiyan, S. A., Croft, B. J., Stringer, J. K., and Deomano, E. C. 2015. Pathogenic variation in spore populations of Sporisorium scitamineum, causal agent of sugarcane smut in Australia. Plant Dis. 99:93-99.

An incursion of sugarcane smut in Queensland was identified in 2006 in Bundaberg, Ingham, and Mackay. In 2008, two cultivars, 'Q205' and 'Q170', were highly susceptible in Bundaberg but remained disease free on a heavily infested farm in Mackay. A glasshouse experiment was established to determine whether the differences in disease expression were due to the sources of the two cultivars sourced from Bundaberg and Mackay in 2009. These were inoculated with the Mackay population of Sporisorium scitamineum spores. These cultivars had the same levels of disease, indicating that the variation in the clonal source of the cultivars was not responsible for the observed differences. A second glasshouse experiment in 2013 confirmed that highly suscepti-

ble Q205 and Q170 were resistant to the Mackay population of $S$. scitamineum but remained susceptible to the Bundaberg population. In 2010, S. scitamineum populations of smut fungi from Mackay and Bundaberg were compared in a field trial and the Mackay population had significantly less disease than the Bundaberg population on four cultivars (Q170, Q205, 'Q174', and 'Q138') but had significantly more disease in 'Q188'. These results confirmed the field observations and suggested that there was a differential response among the cultivars for the smut fungi collected from Mackay compared with Bundaberg and are genetically different. This finding suggests that a mixture of spores should be used to inoculate sugarcane clones for resistance screening.
\end{abstract}

Sugarcane smut, caused by the fungus Sporisorium scitamineum (Syd.) M. Piepenbr., M. Stoll \& Oberw. (formerly called Ustilago scitaminea) (29), is an important disease of sugarcane worldwide (10). It was recorded for the first time in Australia in 1998, when it was found in the Ord River Irrigation Area of Western Australia (12). Smut was reported for the first time in Queensland, the major sugarcane-growing area in Australia, in June 2006 (13). The smut fungus infects plants through buds or germinating shoots and grows in association with the apical and lateral meristems of shoots as they develop (17). The fungus stimulates the production of a characteristic fruiting body known as a sorus, which is a combination of plant and fungal tissues (14). Sori are produced from as early as 6 to 8 weeks after planting or ratooning and continue to be produced throughout the season on new tillers or from side shoots on mature stalks (12). Infected plants become stunted and tend to produce excessive tillering, with up to 50 to 100 tillers per plant, each with a sorus. Ratooning can induce symptom development in latently infected plants (12).

Sugarcane smut can cause significant yield loss if susceptible cultivars are planted (10). In Louisiana in the United States, 0.6 to $0.7 \%$ yield loss was estimated for every $1 \%$ increase of diseased plants (18). In Australia, yield losses up to $62 \%$ were predicted if susceptible cultivars are planted (21).

The most effective way to manage sugarcane smut is through the propagation of resistant cultivars (10). Other control measures that are used in conjunction with resistant cultivars are hot water treatment and fungicide application to seedcane, and roguing of infested stools or plough out of infested crops $(2,7,10)$.

After detection of smut in Queensland in 2006, Sugar Research Australia (SRA, former BSES) commenced a large screening program to develop smut-resistant cultivars in Australia. The main

Corresponding author: S. Bhuiyan,

E-mail: sbhuiyan@sugarresearch.com.au

Accepted for publication 21 June 2014.

http://dx.doi.org/10.1094/PDIS-12-13-1257-RE

(C) 2015 The American Phytopathological Society objectives of this program were to maintain the current rate of genetic gain by doubling the size of the selection program, and to increase the proportion of smut-resistant cultivars in the selection program by making crosses between resistant parents. In screening trials, cultivars from various stages of breeding programs, parents, and foreign clones are screened against sugarcane smut. The stalks of test clones are cut into one-bud setts (cuttings) and then dipped into a smut spore suspension (approximately $5 \times 10^{6}$ spores $/ \mathrm{ml}$ deionized water) for $10 \mathrm{~min}$ and maintained at $31^{\circ} \mathrm{C}$, which is ideal for smut infection and germination of the setts $(5,19)$. Spores of sugarcane smut for the screening trial were collected from smut sori on the susceptible 'Q205' from the SRA smut research farm, Bundaberg (S 25.049023 ${ }^{\circ}$, E $152.3944^{\circ}$ ), Queensland; Q205 has been used as a source of spores for SRA's smut screening program since 2006. This cultivar is included in the smut screening trials as a highly susceptible standard, and typically has approximately 80 to $100 \%$ smut incidence with the dip inoculation and natural infection experiments (6). Another cultivar, 'Q170', was tested over 20 trials, and smut incidence of 50 to $100 \%$ has been recorded.

Recent results from a research trial in Mackay, Central Queensland, had very low or no incidence of smut on two of the susceptible cultivars, Q205 and Q170; whereas, another susceptible cultivar, 'Q157', had very high incidence of smut (22). This experiment was conducted on the farm where smut was first identified in the Mackay region, which is approximately $600 \mathrm{~km}$ north of Bundaberg.

A few possible explanations for the observed differences in the reaction of these susceptible cultivars are as follows. (i) Sugarcane is clonally propagated and rare mutations in cultivars can go undetected, which may indicate that different plant sources can have slight genetic differences $(1,9,23,24)$. The identity of the cultivar in Mackay and Bundaberg was confirmed by DNA fingerprinting but this is unlikely to detect minor genetic changes. (ii) Differences in the environment between the two regions were responsible for the differences in the reactions of the cultivar $(1,4)$. (iii) There were genetic differences in the smut fungus affecting sugarcane in Mackay and Bundaberg $(1,15,16)$.

Strains of $S$. scitamineum causing differences in reaction of cultivars have been reported in a number of countries $(10,12,26)$. Strains or races of smut have been reported in Hawaii, Taiwan, 
Brazil, and Pakistan (14). For a successful management strategy using resistant cultivars, it is important to understand variation of pathogen population. The aims of the study were to determine whether the observed variation of pathogenicity occurring between Bundaberg and Mackay was due to changes in the genetics of Q205 and Q170, environmental factors, or pathogenic variation in the smut fungus.

\section{Materials and Methods}

List of sugarcane cultivars and resistance categories. A list of cultivars used in this study and their resistance categories are presented in Table 1. Except where indicated otherwise, the resistance categories were based on historical smut screening data at SRA, Bundaberg and extensive field observation. Sugarcane cultivars used in this study were classified as highly resistant $=$ these cultivars can be grown in all areas, rarely show smut symptoms (0-3\% infected plants), and do not suffer yield loss; resistant $=$ these cultivars can be grown in all areas, sometimes low levels of smut (4 to $10 \%$ infected plants) symptoms observed, usually do not suffer yield loss; intermediate $=$ these cultivars may show low to medium levels of smut (11 to $20 \%$ infected plants), can be grown in most areas, may suffer yield loss with very high smut disease pressure; susceptible $=$ these varieties will show smut (21 to $30 \%$ infected plants ), may suffer yield loss if planted in the areas that have heavy smut disease pressure or in the areas highly conducive to smut; and highly susceptible $=$ these varieties will show severe smut symptom (30 to $100 \%$ infected plants ) and will suffer severe yield loss.

Evaluation of source of sugarcane. A glasshouse experiment was established at SRA, Central Experiment Station, Mackay, Queensland (S 21.20, E $149.50^{\circ}$ ), to determine whether there was variation within clonal plant sources of Q205 and Q170 from Bundaberg and Mackay, and to determine whether environmental factors could be responsible for the observed difference. At the time this experiment was conducted, it was not possible to move spore collections between regions because of quarantine restrictions.

S. scitamineum spore collection and assessment of germination. Fresh teliospores of S. scitamineum were collected from smut sori on susceptible Q157 from Schembri's farm (where Mackay population of S. scitamineum first reported), in Habana, Mackay (S $21.20^{\circ}, \mathrm{E} 149.50^{\circ}$ ), Queensland (M spore population). Mature sori were collected by cutting the sorus 10 to $20 \mathrm{~cm}$ below the top visible dewlap and placing it in a plastic bag. Spores were collected from the sorus by scraping and subsequent sieving through a nylon net ( 1 by $1 \mathrm{~mm}$ ) to remove plant materials.

Spore suspensions were made by adding $0.1 \mathrm{~g}$ of smut spores to $100 \mathrm{ml}$ of sterile deionized water with a drop of Tween 20 and mixed thoroughly with a magnetic stirrer. The spore concentration

Table 1. List of cultivars and their disease response to sugarcane smut in Australia ${ }^{y}$

\begin{tabular}{lcc}
\hline Cultivar $^{\mathbf{z}}$ & Resistant category & Response in Mackay \\
\hline Q171( $)$ & HR & HR \\
KQ228( & R & $\ldots$ \\
Q200( & R & $\ldots$ \\
Q183( & I & $\ldots$ \\
Q208( & I & $\ldots$ \\
Q190( & I & $\ldots$ \\
Q138 & S & $\ldots$ \\
Q188( & S & $\ldots$ \\
Q170( $*$ & HS & HR \\
Q117 & HS & $\ldots$ \\
Q174( & HS & $\ldots$ \\
Q205( $*$ & HS & HR \\
Q157* & HS & HS \\
\hline
\end{tabular}

${ }^{\mathrm{y}} \mathrm{HR}=$ highly resistant, $\mathrm{R}=$ resistant, $\mathrm{I}=$ intermediate, $\mathrm{S}=$ susceptible, and HS $=$ highly susceptible.

${ }^{z}$ Cultivars followed by asterisks were used in glasshouse experiments and all cultivars except Q157 were used in field experiment; (1) is the plant breeders' right (PBR) symbol for Australian cultivars. of the suspension was determined with a hemocytometer and adjusted to $1.5 \times 10^{6}$ spores $\mathrm{ml}^{-1}$. Viability of inoculum was monitored and only spore suspensions with $>90 \%$ spore viability were used.

Treatments. Four cultivars with a range of resistance to smut were included in the experiment, including Q170 and Q205, which had shown different field reactions to smut between Mackay and Bundaberg (Table 1). Each variety was sourced from plots that had been verified to be the correct variety by DNA fingerprinting in low smut-incidence areas in Mackay and Bundaberg.

Inoculation. Two methods of inoculation were used: dip and injection. Stalks of sugarcane cultivars were stripped to expose the bud, then cut into one-bud setts using an electric saw. For the dip inoculation, setts were placed in mesh bags and submerged in a smut spore suspension (approximately $1.5 \times 10^{6} \mathrm{spores} / \mathrm{ml}$ of deionized water) for $10 \mathrm{~min}$ before planting in peat pots. For the injection method, setts were inoculated with smut spore suspension by injecting a small amount of a smut spore suspension at the base of the bud with a hypodermic syringe. A set of noninoculated setts from each cultivar was included in this experiment as a control.

Trial establishment and trial design. The experiment was established on 10 March 2009. Inoculated setts were planted in peat pots filled with potting mix, watered up to saturation, and transferred to a polytunnel greenhouse. Plants were irrigated using an automated irrigation system one to two times a day, depending on weather conditions. Plants were fertilized as required. Temperature and relative humidity inside the polytunnel were recorded.

The experiment was set out in a randomized complete block design with three replicates and 10 plants in each replication.

Disease assessment. Disease was assessed after 6 weeks as soon as the first smut sorus was detected; then, the trial was inspected every week. Diseased plants were removed from the polytunnel as soon as sori of smut were detected to stop cross contamination. The trial was finished when no new symptoms of smut were observed for 12 months. Disease assessment in each replication was made by counting the total number of plants and the number of plants showing smut sori. Percentage of disease incidence (DI) was calculated as DI $=(I / T) \times 100$, where $I=$ number of plants infected and $T=$ total number of plants counted.

Evaluation of $\boldsymbol{S}$. scitamineum populations. A glasshouse experiment was established at SRA, Woodford Pathology farm, Queensland (S 26.93 ${ }^{\circ}$ E $152.78^{\circ}$ ) in June 2013 to determine whether there were differences in virulence among Bundaberg (B) and M S. scitamineum populations.

S. scitamineum spore populations. Two spore populations of $S$. scitamineum were collected from the field at Mackay and Bundaberg. For the M spore population, smut sori were collected by Dr. George Piperidis in early June 2013 from Q157 from the Shembri's farm in Habana, Mackay. The B spore population was collected from SRA smut research farm, Bundaberg from Q205 in January 2013. The B populations of $S$. scitamineum has been maintained on Q205 for routine smut screening trials. Spore collection and processing procedures were same as described earlier.

Treatments. Four cultivars used in glasshouse experiment 1 were included in this experiment, including Q170 and Q205, which had shown differential field reactions to smut spore populations sourced from Mackay and Bundaberg (Table 1).

Inoculation. Stalks of sugarcane cultivars were stripped to expose the bud, then cut into one-bud setts using an electric saw. One-bud setts were inoculated using the procedures described earlier. A set of uninoculated setts from each cultivar was included in this experiment as a control.

Trial design. The experiment was set out in a factorial design with two main effects (spore population and cultivar), with five replicates and three plants in each replication.

Disease assessment. Disease was assessed after 8 weeks, as soon as the first smut sorus was detected; then, the trial was inspected every week. Diseased plants were removed from the glasshouse as soon as the symptom of smut was detected to stop cross contamination. Percentage of infection (DI) was calculated as described in previous experiment. 
Field experiment using $\mathrm{B}$ and $\mathrm{M}$ populations of $S$. scitamineum. The experiment was established at SRA, Woodford Pathology farm, Queensland in July 2010. Woodford was selected for this experiment because it had very low levels of natural infection with smut at the time of planting the experiment. Twelve cultivars from various resistance categories were used in this trial (Table 1). Cultivars used in this experiment were planted after hot-water $\left(50^{\circ} \mathrm{C} 2\right.$ h) and fungicide (propiconazole) treatment in an area $20 \mathrm{~km}$ from commercial sugarcane crops. Whole stalks were collected and sent to Woodford prior to commencement of the experiment.

S. scitamineum spore populations. For the $\mathrm{M}$ spore population, six cardboard boxes, each containing approximately 10 to 15 smut sori, were collected from Schembri's farm from Q157 at Habana Mackay, where the M population of S. scitamineum was detected. The B spore population was collected from SRA Bundaberg smut research farm from Q205 in May 2010.

Spore collection and processing procedures were the same as described earlier. After collection, spores were maintained in sealed containers at $5^{\circ} \mathrm{C}$ until use. To assess viability, germination of teliospores of each spore population was assessed on $1.5 \%$ water agar after incubation at $31^{\circ} \mathrm{C}$ for $6 \mathrm{~h}$ before inoculation.

Inoculation, planting, and trial design. Inoculation of test cultivars with smut spores was carried out on 14 July 2010. Stalks of the test cultivars were cut into one-bud setts using an electric saw and the setts were either dipped into smut spore suspensions (approximately $5 \times 10^{6}$ spores $/ \mathrm{ml}$ of deionized water) of spore population $\mathrm{M}$ or $\mathrm{B}$ for $10 \mathrm{~min}$. Setts were then maintained in separate plastic trays in moist vermiculite and kept at $31^{\circ} \mathrm{C}$ and $>80 \%$ relative humidity (19) for 7 days. Germinated setts were then planted in peat pots and kept in a polytunnel greenhouse for approximately 3 months before transplanting to the field in November 2010. Irrigation, fertilizer application, and insecticide and herbicide treatments were done according to normal farm practices.

The trial was planted using a randomized complete block design, with five replications and eight plants in each replicate.

Disease assessment and data analysis. Inspection for smut was conducted in the polytunnel and in the field every week after the first smut sorus was detected on 29 September 2010. The infected plants were removed from the trial as soon as they were detected to reduce the chance of cross contamination. Observations ceased on 27 May 2011. Our observation and available literature suggested that the latent period and symptom expression time of sugarcane smut could be 3 to 6 months, depending on cultivar and environmental condition (31). To minimize the chance of secondary infec-

Table 2. Incidence of smut on sugarcane cultivars planted from clonal planting material originating from Bundaberg and Mackay and following inoculation by two methods with sugarcane smut spores collected from Habana, Mackay (M)

\begin{tabular}{lcc}
\hline & \multicolumn{2}{c}{ Source $^{\mathbf{z}}$} \\
\cline { 2 - 3 } Inoculation method, cultivar & Bundaberg & Mackay \\
\hline Dip & $21.8 \mathrm{c}$ & $29.4 \mathrm{c}$ \\
Q157 & $0.0 \mathrm{e}$ & $0.0 \mathrm{e}$ \\
Q205 & $0.0 \mathrm{e}$ & $0.0 \mathrm{e}$ \\
Q170 & $0.0 \mathrm{e}$ & $0.0 \mathrm{e}$ \\
Q171 & & \\
Inject & $89.2 \mathrm{a}$ & $90.9 \mathrm{a}$ \\
Q157 & $45.1 \mathrm{~b}$ & $48.5 \mathrm{~b}$ \\
Q205 & $6.5 \mathrm{de}$ & $17.3 \mathrm{~cd}$ \\
Q170 & $0.0 \mathrm{e}$ & $0.0 \mathrm{e}$ \\
Q171 & & \\
Control & $0.0 \mathrm{e}$ & $3.0 \mathrm{de}$ \\
Q157 & $0.0 \mathrm{e}$ & $0.0 \mathrm{e}$ \\
Q205 & $0.0 \mathrm{e}$ & $0.0 \mathrm{e}$ \\
Q170 & $0.0 \mathrm{e}$ & $0.0 \mathrm{e}$ \\
Q171 &
\end{tabular}

${ }^{\mathrm{z}}$ Each value is the mean of three replications, with 10 plants per replication. Means followed by with the same letters in column or row are not significantly different using Fisher's least significance difference test $(P<$ $0.05)$. Analysis was performed on logit-transformed data. tion and cross contamination between spore populations, data obtained until 21 December 2010 (161 days or 5 months after inoculation) were used in the results. Assessment was made by counting the total number of plants and the number of plants showing sori. From these numbers, cumulative percent incidence of the smut per plot was calculated. A logit-transformation was applied prior to analysis (33): logit proportion $=\log [$ (total diseased plants + $0.5) /($ total plants - total diseased plants +0.5$)]$.

The SAS Proc mixed procedure was used to analyze data (version 9.2; SAS Institute, Cary, NC), where cultivar, spore population, and disease development time were fixed effects and replicates were random effects. In addition, the correlation between successive times was taken into account in the linear model. The differences of DI between spore populations within a cultivar were calculated using Fisher's protected least significance test. A logistic model was fitted to most of the cultivars to describe the disease progress over time using the SAS Proc nlin procedure. The model was calculated as $I=\{m /(1+\exp [-k($ time $-t m)])\}$, where $I$ = estimated percent DI, $m=$ estimated maximum incidence of smut, $k=$ estimated rate of disease progress, time $=$ day after first inspection, and $\mathrm{tm}=$ inflection point or time (days) required to reach $50 \%$ of the DI (20). Standard errors were calculated using the means and covariance matrix of the estimates, and $t$ tests were performed to compare the estimated parameter between two spore populations.

The curve could not be fitted for five cultivars inoculated with both types of spore population, such as 'KQ228', 'Q171', 'Q200', 'Q190', and 'Q208'; and one cultivar, 'Q138', inoculated with the $\mathrm{M}$ spore population, because very little disease was observed.

\section{Results}

Evaluation of source of sugarcane. In this experiment, the control plants were smut free except for one infected plant in Q157 from Mackay 5 months after inoculation (31 July), which showed that there was little transmission between plants in the polytunnel. No disease was observed in dip-inoculated Q170, Q171, or Q205 but Q157 had more than 20\% infection in both the Mackay and Bundaberg cultivar sources (Table 2). In the plants inoculated by injection, Q157 had 90\% infection in plants sourced from both Mackay and Bundaberg (Table 2). For plants sourced from Mackay and Bundaberg, there were similar levels of infection on Q205 (45 and $48 \%$, respectively); Q170 had 6 and 17\% incidence, respectively; and Q171 had no disease. Highest smut incidences (89 and 90\%) were observed in Q157. There was no significant difference between the sources of the clonal materials used for planting the trial.

Evaluation of $S$. scitamineum populations-glasshouse experiment. The control (noninoculated) plants remained smut free, indicating no cross contamination within the experiment. Significantly higher levels of smut infection were observed on highly susceptible Q205 (93\%) and Q170 (47\%) inoculated with the B spore population compared with very little or no smut incidence observed on these cultivars (6.7 and $0 \%$, respectively) when inoculated with the $\mathrm{M}$ spore population (Table 3). High incidence of smut was observed in Q157 when inoculated using M or B spore populations, although significantly higher incidence was observed

Table 3. Incidence of smut on sugarcane cultivars inoculated with Bundaberg (B) and Mackay (M) spore populations

\begin{tabular}{lccc}
\hline & \multicolumn{3}{c}{ Spore populations $^{\mathbf{z}}$} \\
\cline { 2 - 4 } Cultivar & $\mathbf{B}$ & $\mathbf{M}$ & Control \\
\hline Q250(b) & $93.3 \mathrm{a}$ & $6.7 \mathrm{~cd}$ & $0.0 \mathrm{~d}$ \\
Q170( & $46.7 \mathrm{~b}$ & $0.0 \mathrm{~cd}$ & $0.0 \mathrm{~d}$ \\
Q157 & $25.0 \mathrm{bc}$ & $66.7 \mathrm{a}$ & $0.0 \mathrm{~d}$ \\
Q171(b) & $0.0 \mathrm{~d}$ & $0.0 \mathrm{~d}$ & $0.0 \mathrm{~d}$ \\
\hline
\end{tabular}

${ }^{\mathrm{z}}$ Each value is the mean of five replications, with three plants per replication. Means followed by with the same letters in column or row are not significantly different using Fisher's least significance difference test $(P<$ $0.05)$. Control $=$ no inoculation. Analysis was performed on logittransformed data. 
in plants inoculated using the $M$ spore population (66.7\%) compared with plants inoculated using the B spore population $(25 \%)$. No smut developed on the highly resistant Q171 when inoculated with the $\mathrm{B}$ or $\mathrm{M}$ spore population. This result confirmed that the observed differences in reaction of Q205 and Q170 were due to differences in pathogenic variability related to cultivar within $S$. scitamineum.

Evaluation of $S$. scitamineum populations-field experiment. Significant effects of three-way and two-way interactions were detected between spore populations, cultivars, and disease development time (Table 4) in the field experiment at Woodford. Disease data from the final inspection are presented in Table 5. By 161 days of the experiment, susceptible Q170, 'Q174', Q205, and Q138 had significantly higher incidence of smut when inoculated with the B spore population compared with the $\mathrm{M}$ spore population (Table 5). However, susceptible 'Q188' had significantly higher smut incidence when inoculated with the $\mathrm{M}$ spore population than the B spore population. There were no significant differences in smut incidence between spore populations in the intermediate and resistant cultivars.

Disease progress over time within a cultivar after inoculation with the B or M spore population of $S$. scitamineum is shown in Figure 1. Out of six cultivars, differential trends of disease progress were observed in Q205, Q188, Q174, Q170, and Q138 when inoculated with the different spore populations.

In highly susceptible Q205, the first disease was observed for the B spore population after 77 days of inoculation, whereas it took 103 days to show any disease for the M spore population (Fig. 1A). The rate of disease progress was faster with the B spore population compared with spore population $\mathrm{M}$, and the time to reach $50 \%$ of predicted maximum disease level was estimated at 49 and 101 days for spore populations from Bundaberg and Mackay, respectively (Table 6). A comparison of estimated values of maximum disease $(m)$, rate of disease progress $(k)$, and time to reach estimated $50 \%$ maximum disease level $(\mathrm{tm})$ using a $t$ test indicated that there were highly significant $(P<0.0001)$ differences in the disease progress in Q205 between spore populations from Bundaberg and Mackay (Table 6).

Contrasting responses were observed in Q188, where the first smut was observed after 133 days of inoculation for the $M$ spore population, which reached maximum (55\%) at 161 days (Fig. 1B). In contrast, for the B spore population, the first smut was detected at 139 days after inoculation and reached a maximum (30\%) after 161 days of inoculation. No significant differences were observed between spore populations $\mathrm{B}$ and $\mathrm{M}$ in relation to rate of disease progress $(k)$ and estimated days to reach $50 \%$ maximum disease level $(\mathrm{tm})$ (Table 6).

There were no significant differences in the rate of disease development or median disease progress time for Q174 and Q170 between the two spore populations, even though the maximum DIs were different (Fig. 1C and D; Table 6). Over 17\% disease was observed in Q138 after 161 days of inoculation by the B spore population, whereas no disease was detected when inoculated with the $\mathrm{M}$ spore population during this time (Fig. 1E). No significant differences in disease development in 'Q117' were observed between the two spore populations (Fig. 1F).

\section{Discussion}

After the first incursion of smut in Queensland, the main sugarcane-growing area, in 2006, a concerted effort was made to in- crease smut-resistant cultivars through breeding for the Australian sugar industry. In this process, the field performances of current cultivars and advanced lines were tested in various growing regions under natural infection of smut. A few of these cultivars exhibited resistance in the Mackay growing region but susceptibility in the Bundaberg growing region. This study examined whether the differences were due to genetic changes in the cultivars or due to the pathogenic variability within regional $S$. scitmineum populations.

The results from the first glasshouse trial at Mackay showed that there was no difference between Q205 and Q170 sourced from Bundaberg or Mackay. These cultivars were still more resistant to the smut collected from the Mackay site, even when inoculated under standard conditions and grown in an artificial potting mix in the greenhouse, which suggests that environment is not a factor in the different smut resistance of these cultivars at Mackay.

Results from the second glasshouse experiment indicated that the M spore population of $S$. scitamineum was different from the B spore population in regards to their pathogenicity on Q205 and Q170. These results are in agreement with Magarey et. al. (22), who suggested that Q205 and Q170 were more resistant to smut spores collected from Habana in Mackay than when they were screened for resistance in Bundaberg. These results also suggest that environment was not a factor for the difference in expression of resistance.

A similar finding occurred in the field experiment, because Q205, Q170, and Q174 had higher incidence of smut when inoculated with the B compared with M spore population. Q188 showed an opposite reaction, being more susceptible to the $\mathrm{M}$ smut population compared with the B smut population. Q188 is not grown commercially in Mackay. Overall, the results from this study confirmed the existence of pathogenic variability in the S. scitamineum population interacting with sugarcane in Australia. This is the first report of variability of sugarcane smut virulence in Australia.

Pathogenic races of $S$. scitamineum have been reported in many countries. A new race of smut appeared in Hawaii in 1976, only 5 years after the first detection of smut in 1971, and again in 2001 $(11,27)$. Races also reported in Taiwan, Pakistan, Nigeria, and Kenya $(12,25,32)$. Although races of sugarcane smut were reported

Table 5. Incidence (\%) of smut after 161 days of inoculation on cultivars inoculated with Bundaberg (B) and Mackay (M) spore populations of Sporisorium scitamineum in a field experiment at Woodford, Queensland

\begin{tabular}{lccc}
\hline & \multicolumn{2}{c}{ Smut spore population } & \\
\cline { 2 - 3 } Cultivar & B & M & Significance $^{\mathbf{z}}$ \\
\hline KQ228 & 7.5 & 0 & $\mathrm{~ns}$ \\
Q117 & 45.5 & 52.6 & $\mathrm{~ns}$ \\
Q138 & 17.5 & 0 & $*$ \\
Q170 & 30 & 15 & $*$ \\
Q171 & 0 & 0 & $\mathrm{~ns}$ \\
Q174 & 37.5 & 17.5 & $*$ \\
Q183 & 2.5 & 2.5 & $\mathrm{~ns}$ \\
Q188 & 30 & 55 & $*$ \\
Q190 & 0 & 7.5 & $\mathrm{~ns}$ \\
Q200 & 5 & 0 & $\mathrm{~ns}$ \\
Q205 & 42.5 & 7.5 & $*$ \\
Q208 & 0 & 0 & $\mathrm{~ns}$
\end{tabular}

${ }^{\mathrm{z}}$ Symbols: $\mathrm{ns}=$ not significant and $*=$ significantly different using Fisher's least significance test $(P<0.05)$.

Table 4. Analysis of variance for the field experiment, spore populations, cultivars, and disease development time and their interactions

\begin{tabular}{|c|c|c|c|c|}
\hline Effects & Numerator df & Denominator df & $F$ value & $P>F$ \\
\hline Spore populations (SP) & 1 & 129 & 3.86 & 0.0516 \\
\hline Cultivar (C) & 11 & 129 & 20.34 & $<0.0001$ \\
\hline $\mathrm{SP} \times \mathrm{C}$ & 11 & 129 & 2.73 & 0.0033 \\
\hline Disease development time (DT) & 22 & 2,103 & 27.73 & $<0.0001$ \\
\hline $\mathrm{SP} \times \mathrm{DT}$ & 22 & 2,103 & 2.24 & 0.0008 \\
\hline $\mathrm{C} \times \mathrm{DT}$ & 242 & 2,066 & 4.08 & $<0.0001$ \\
\hline $\mathrm{SP} \times \mathrm{C} \times \mathrm{DT}$ & 242 & 2,066 & 1.29 & $<0.0032$ \\
\hline
\end{tabular}


in most of the major sugarcane-growing countries, Ferreira and Comstock (14) considered the true prevalence of races to be controversial. Variable smut reactions have been reported for the same cultivars in different countries, and some of those might be attributed to the differences in inoculation methods. Two international collaborations have attempted to standardize race typing. Gillaspie et al. (15) conducted a trial for race identification under glasshouse conditions and determined six races. Grisham (16) coordinated a race typing study in 10 countries using local spore populations tested against a standardized set of 11 differential cultivars. Although pathogenic variation throughout the world was observed, the reaction of the common set of cultivars to the local populations was similar at all locations, except Taiwan. The results suggested the existence of a different race at that location. There have been no previous reports of variation in smut virulence in Australia but this is understandable, given the short period of time the disease has been present.
Commercial sugarcane cultivars are polyploidy hybrids of various Saccharum spp. The lack of clearly defined races of sugarcane smut could be due to characteristics of sugarcane as a crop plant. Smut resistance is a multifactorial process determined by combination of structural and chemical barriers, and is induced by a number of resistance genes $(28,30,34)$. These features have resulted in very few strict gene-for-gene relationships, and resistance to most sugarcane diseases is quantitative (28).

The implication of the difference in virulence of the $\mathrm{M}$ and $\mathrm{B}$ smut spore populations to our breeding program is not clear. Fortunately, in this experiment, no resistant or intermediate cultivars showed susceptible reactions to either spore population. Smut spores can spread long distances and we believe that, if there were difference between regions, they would disappear, because mixing of smut spores between regions does occur (12). In fact, high levels of smut are now being reported in sugarcane clone Q205 from most parts for the Mackay region, although, the glasshouse exper-
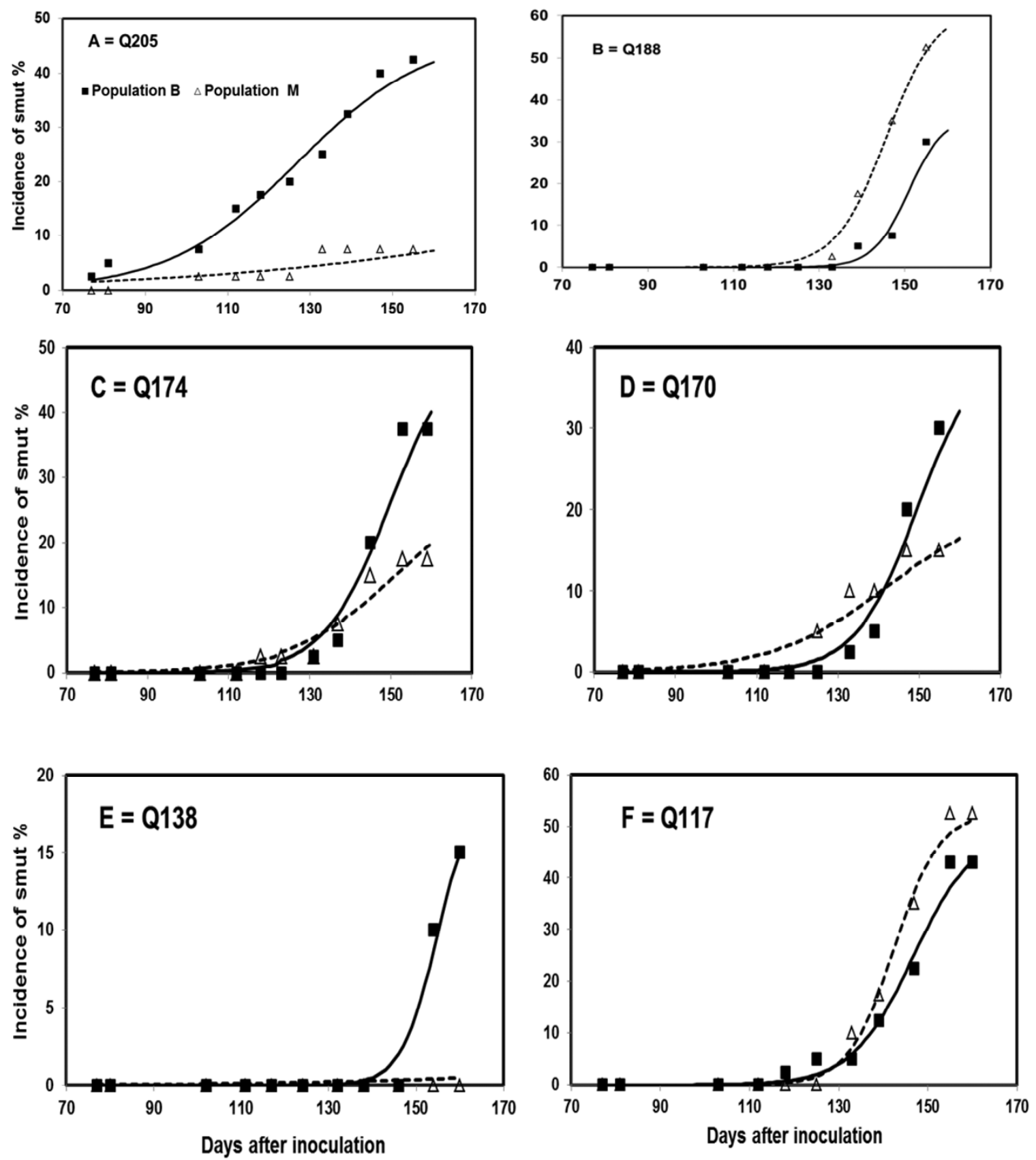

Fig. 1. Actual and estimated progress of disease over time on cultivars inoculated with Bundaberg (B) and Mackay (M) spore populations of Sporisorium scitamineum in 2010 at the Sugar Research Australia Woodford pathology farm, Queensland. 
iment 2 confirmed the existence of that spore populations in the Habana area, at least until June 2013.

One possible explanation for the variation in the smut virulence at the two sites is that the spores that initiated the incursions at these sites may have been slightly different. This may have resulted in a founder effect (23), which is defined as the loss of genetic variation that occurs when a new population is established by a very small number of individuals from a larger population, resulting in the new population being distinctively different, both genetically and phenotypically, from the parent population. Braithwaite et al. (8) compared S. scitamineum isolates from the Ord River Irrigation Area (ORIA) Western Australia, Asia, Africa, and the United States using DNA fingerprinting, and speculated that the most likely source of a smut incursion to eastern Australia would be either from Indonesia or the ORIA, which were genetically similar. However, they also detected distinctive isolates in other parts of Asia. They suggested that it is essential to obtain pathogenicity data to determine whether these could constitute a disease threat to the Australian sugar industry. Croft et al. (13) suggested that the incursions in Bundaberg and Mackay regions occurred at the same time, possibly from the same weather event blowing spores from the Ord River in Western Australia. The small number of spores that landed in Mackay could have been slightly different from those in Bundaberg. Another factor which also makes this

Table 6. Estimated parameters and associated statistics for the model to describe the disease progress over time after inoculation of sugarcane cultivars with Bundaberg (B) and Mackay (M) spore population of Sporisorium scitamineum

\begin{tabular}{|c|c|c|c|}
\hline \multirow[b]{2}{*}{ Cultivar ${ }^{\mathrm{z}}$} & \multicolumn{2}{|c|}{ Smut spore population } & \multirow[b]{2}{*}{$P$ value } \\
\hline & B & M & \\
\hline \multicolumn{4}{|l|}{ Q205 } \\
\hline$m$ & $46.33( \pm 2.58)$ & $19.18( \pm 7.6)$ & $<0.0001$ \\
\hline$k$ & $0.07( \pm 0.01)$ & $0.02( \pm 0.01)$ & $<0.0001$ \\
\hline $\mathrm{tm}$ & $49.27( \pm 3.2)$ & $100.9( \pm 44.15)$ & $<0.0001$ \\
\hline$R^{2}$ & 92.11 & 80.29 & $\ldots$ \\
\hline RMSE & 11.41 & 7.36 & $\ldots$ \\
\hline \multicolumn{4}{|l|}{ Q188 } \\
\hline$m$ & $33.95( \pm 2.65)$ & $61.06( \pm 5.09)$ & $<0.0001$ \\
\hline$k$ & $0 . .33( \pm 0.18)$ & $0.18( \pm 0.08)$ & 0.64 \\
\hline $\mathrm{tm}$ & $73.15( \pm 2.4)$ & $68.02( \pm 3.07)$ & 0.22 \\
\hline$R^{2}$ & 78.00 & 76.80 & $\ldots$ \\
\hline RMSE & 14.97 & 26.87 & $\ldots$ \\
\hline \multicolumn{4}{|l|}{ Q174 } \\
\hline$m$ & $42.96( \pm 2.98)$ & $28.95( \pm 4.03)$ & $<0.0001$ \\
\hline$k$ & $0.208( \pm 0.08)$ & $0.08( \pm 0.04)$ & 0.32 \\
\hline$t m$ & $70.66( \pm 2.34)$ & $74.84( \pm 7.31)$ & 0.74 \\
\hline$R^{2}$ & 88.70 & 67.15 & $\ldots$ \\
\hline RMSE & 13.97 & 16.12 & $\ldots$ \\
\hline \multicolumn{4}{|l|}{ Q170 } \\
\hline$m$ & $35.96( \pm 3.17)$ & $17.8( \pm 3.04)$ & $<0.0001$ \\
\hline$k$ & $0.179( \pm 0.08)$ & $0.11( \pm 0.08)$ & 0.39 \\
\hline$t m$ & $69.93( \pm 3.19)$ & $58.08( \pm 8.22)$ & 0.47 \\
\hline$R^{2}$ & 75.12 & 52.17 & $\ldots$ \\
\hline RMSE & 17.71 & 15.80 & $\ldots$ \\
\hline \multicolumn{4}{|l|}{ Q138 } \\
\hline$m$ & $15.55( \pm 1.62)$ & $\ldots$ & $\ldots$ \\
\hline$k$ & $0.59( \pm 1.49)$ & $\ldots$ & $\ldots$ \\
\hline $\mathrm{tm}$ & $77( \pm 2.86)$ & $\ldots$ & $\ldots$ \\
\hline$R^{2}$ & 64.16 & $\ldots$ & $\ldots$ \\
\hline RMSE & 10.84 & $\ldots$ & $\ldots$ \\
\hline \multicolumn{4}{|l|}{ Q117 } \\
\hline$m$ & $45.61( \pm 3.9)$ & $53.24( \pm 1.7)$ & 0.22 \\
\hline$k$ & $0.17( \pm 0.08)$ & $0.19( \pm 0.04)$ & 0.44 \\
\hline$t m$ & $68.27( \pm 3.3)$ & $65.57( \pm 1.2)$ & 0.20 \\
\hline$R^{2}$ & 81.18 & 96.03 & $\ldots$ \\
\hline RMSE & 18.62 & 8.61 & $\ldots$ \\
\hline
\end{tabular}

${ }^{\mathrm{z}}$ Parameter: $m=$ estimated maximum incidence of smut, $k=$ estimated rate of disease development, and $t m=$ inflection point or time (days) take to reach $50 \%$ of maximum disease level. Values in parentheses are asymptotic standard error; $R^{2}=1$ - residual Sum of squares (SS)/regression SS; root mean squares error $($ RMSE $)=\sqrt{ }($ residual SS/number of observations). complicated is that sugarcane smut fungus as well as other smut fungi can readily hybridize between races and even between species (3).

Resistance screening for sugarcane smut by artificial inoculation method or natural infection should be carried out in different geographical location to identify the new biotypes or races into different geographical regions. Effort should be made to use a mixture of spores collected from various geographical regions for artificial inoculation in future screening trials. Nevertheless, the Australian sugar industry needs to be prepared for possible loss of resistance of cultivars to sugarcane smut.

\section{Acknowledgments}

Australian Sugar Research and Development Corporation (SRDC) partly funded this project. We thank G. Piperidis for support; I. Mendezona, G. Bade, B. Rae, L. Meagher, and P. Wickramasinghe for technical assistance; and the senior editor and anonymous reviewers for valuable criticism and suggestions.

\section{Literature Cited}

1. Arnold, M. H., and Brown, S. J. 1968. Variation in the host-parasite relationship of a crop disease. J. Agric. Sci. 71:19-36.

2. Bailey, R. 1980. Possibilities for the control of sugarcane smut (Ustilago scitaminea) with fungicides. South Afr. Sugar J. 1980:158-164.

3. Bakkeren, G., and Kronstad, J. W. 1996. The pheromone cell signalling components of the Ustilago a mating-type loci determine intercompatibility between species. Genetics 143:1601-1613.

4. Barnett, H. L., and Binder F. L. 1973. The fungal host-parasite relationship. Rev. Phytopathol. 11:273-292.

5. Bhuiyan, S. A., Croft, B. J., Cox, M. C., and Bade, G. 2009. Some biological parameters of the sugarcane smut fungus, Ustilago scitaminea. Proc. Aust. Soc. Sugar Cane Technol. 31:125-134.

6. Bhuiyan, S. A., Croft, B. J., Cox, M. C., and Bade, G. 2010. Varietal resistance of sugarcane to natural infection of smut-preliminary results. Proc. Aust. Soc. Sugar Cane Technol. 32:355-365.

7. Bhuiyan, S. A., Croft, B. J., James, R. S., and Cox, M. C. 2012. Laboratory and field evaluation of fungicides for the management of sugarcane smut caused by Sporisorium scitamineum in seedcane. Australas. Plant Pathol. 41:591-599.

8. Braithwaite, K. S., Bakkeren, G., Croft, B. J., and Brumbley, S. M. 2004. Genetic variation in a worldwide collection of the sugarcane smut fungus Ustilago scitaminea. Proc. Aust. Soc. Sugar Cane Technol. 26:48. (CDROM)

9. Burner, D. M., and Legendre, B. L. 1998. Agronomic performance and transmission of the multiple-bud mutation of sugarcane. Crop Sci. 38:928 932.

10. Comstock, J. C. 2000. Smut. Pages 181-185 in: A Guide to Sugarcane Diseases. P. Rott, R. A. Bailey, J. C. Comstock, B. J. Croft, and A. S. Saumtally, eds. CIRAD and ISSCT, Montpellier, France.

11. Comstock, J. C., and Heinz, D. J. 1977. A new race of culmicolous smut of sugarcane in Hawaii. Sugarcane Pathol. Newsl. 19:24-25.

12. Croft, B. J., and Braithwaite, K. S. 2006. Management of an incursion of sugarcane smut in Australia. Australas. Plant Pathol. 35:113-122.

13. Croft, B. J., Magarey, R. C., Allsopp, P. G., Cox, M. C., Willcox, T. G. Milford, B. J., and Wallis, E. S. 2008. Sugarcane smut in Queensland: Arrival and emergency response. Australas. Plant Pathol. 37:26-34.

14. Ferreira, S. A., and Comstock, J. C. 1989. Smut. Pages 211-229 in: Diseases of Sugarcane. C. Ricaud, B. T. Egan, A. G. Gillaspie, Jr., and G. Hughes, eds. Elsevier, Amsterdam.

15. Gillaspie, A. G., Jr., Mock, R. G., and Dean, J. L. 1983. Differentiation of Ustilago scitaminea surrounding area or to the Beltsville spore collections in greenhouse tests. Plant Dis. 67:373-375.

16. Grisham, M. P. 2001. An international project on genetic variability within sugarcane smut. Proc. Int. Soc. Sugar Cane Technol. 24:459-461.

17. Hoy, J. W. 1993. Sugarcane smut in Louisiana: Biology and control. Bull. La. Agric. Exp. Stn. USA

18. Hoy, J. W., Hollier, C. A., Fontenot, D. B., and Grelen, L. B. 1986. Incidence of sugarcane smut in Louisiana and its effect on yield. Plant Dis. 70:59-60

19. Lee-Lovick, G. 1978. Smut of sugarcane-Ustilago scitaminea. Rev. Plant Pathol. 57:181-188.

20. Madden, L. V., Hughes, G., and van den Bosch, F. 2007. The Study of Plant Disease Epidemics. American Phytopathological Society, St. Paul, MN.

21. Magarey, R. C., Bull, J. I., Sheahan T., and Denney, D. 2010. Yield losses caused by sugarcane smut in several crops in Queensland. Proc. Aust. Soc. Sugar Cane Technol. 32:347-354.

22. Magarey, R. C., Denney, D., Sheahan, T., Fowell, L., Croft, B. J., Lonie, K. J., Bull, J. I., Bhuiyan, S., and Willcox, T. G. 2009. Results from smut epidemiology studies in the Herbert, Mackay and Bundaberg-Isis areas in 2007-2008. Proc. Aust. Soc. Sugar Cane Technol. 31:145-157.

23. Mayr, E. 1954. Change of genetic environment and evolution. Pages 157 - 
180 in: Evolution as a Process J. Huxley, A. C. Hardy, and E. B. Ford, eds. Allen and Unwin, London.

24. Nei, M., and Nozawa, M. 2011. Roles of mutation and selection in speciation: from Hugo de Vries to the modern genomic era. Genome Biol. Evol. 3:812-829.

25. Nzioki, H. S., Jamoza, J. E., Olweny, C. O., and Rono, J. K. 2010. Characterization of physiologic races of sugarcane smut (Ustilago scitaminea) in Kenya. Afr. J. Microbiol. Res. 4:1694-1697.

26. Rago, A. M., Casagrande, M. V., and Massola, N. S., Jr. 2009. Variabilidade patogenica de Ustilago scitaminea no Estado de Sao Paulo. Summa Phytopthol. 35:93-97. Mycosphaerella fijiensis. Mol. Ecol. 13:471-482.

27. Schenck, S. 2003. New race of sugarcane smut on Maui. Hawaii Agric. Res. Center-Pathol. Rep. 69:1-4.

28. Schenck, S., Pearl, H. M., Liu, Z., Moore, P. H., and Ming, R. 2005. Genetic variation of Ustilago scitaminea pathotypes in Hawaii evaluated by host range and AFLP markers. Sugarcane Int. 23:15-19.

29. Stoll, M., Piepenbring, M., Begerow, D., and Oberwinkler, F. 2003. Molecular phylogeny of Ustilago and Sporisorium species (Basidiomycota, Us- tilaginales) based on internal transcribed spacer (ITS) sequences. Can. J. Bot. 81:976-984.

30. Wanderley-Nogueira, A. C., Kido, E. A., Soares-Cavalcanti, N. da M., Belarmino, L. C., Bezerra-Neto, J. P., Burnquist, W. L., Chabregas, S. M. Baldani, J. I., and Benko-Iseppon, A. M. 2012. Insight on pathogen defense mechanisms in the sugarcane transcriptome. Funct. Plant Sci. Biotechnol. 6:134-148.

31. Waller, J. J. 1969. Sugarcane smut (Ustilago scitaminea) in Kenya. I. epidemiology. Trans. Br. Mycol. Soc. 52:139-148.

32. Whittle, A. M. 1978. Thoughts on smut resistance testing. Sugarcane Pathol. Newsl. 20:43-46.

33. Xu, X. M., and Ridout, M. S. 1998. Effects of initial epidemic conditions, sporulation rate, and spore dispersal gradient on the spatio-temporal dynamics of plant disease epidemics. Phytopathology 88:1000-1012.

34. You-Xiong, Q., Jian-Wei, L., Xian-Xian, S., Li-Ping, X., and Ru-Kai, C. 2011. Differential gene expression in sugarcane in response to challenge by fungal pathogen Ustilago scitaminea revealed by cDNA-AFLP. J. Biomed. Biotechnol. doi:10.1155/2011/160934 